\title{
Control system design of an intelligent food delivery robot
}

\author{
Haixia Zeng ${ }^{1, a}$, Ziqing Zhang ${ }^{1, b^{*}}$, and Yan Hong ${ }^{1, c}$ \\ ${ }^{1}$ Mechanical \& Electronic Engineering Division, WenHua College, Wuhan, Hubei, 430074, China
}

\begin{abstract}
Contemporary many young people more and more choose to enjoy a variety of food outside, followed by a variety of food service industry emerging in an endless stream of new ideas, new changes. Nowadays, more and more intelligent food delivery robots are appearing in restaurants in many cities, making great contributions to the improvement of the cost and efficiency of food delivery in the catering industry. This paper designs a low-cost, efficient and intelligent food delivery robot, which can receive background instructions, choose the route and order for automatic food delivery, and automatically return after the task. The intelligent food delivery robot adopts a modular design method, mainly including power supply, microprocessor, the keys, drive, positioning, tracking navigation, obstacle avoidance and Wi-Fi communication parts.
\end{abstract}

\section{Introduction}

Due to the rapid development of related technology, in the field of a lot of work, the robots gradually replace humans, help mankind to solve various problems, in the food industry, intelligent food delivery robot is emerging. This paper designs a control system of the intelligent food delivery robot. The idea of modularization is adopted, and the target location of robot is realized through radio frequency module; obstacle avoidance is realized by controlling ultrasonic wave and infrared wave; through the infrared tracking mode to make the robot in accordance with the predetermined route; by controlling PWM, the robot can change speed in the process of motion; confirm to take food through a separate button; through the Wi-Fi module to achieve communication with the background.

\section{Overall design scheme}

In this paper, the overall design scheme of the intelligent food delivery robot is shown in figure 1 . With the microprocessor as the core, the robot is driven by two DC motors to move, and the target identification and positioning are carried out through radio frequency technology, infrared navigation is adopted, ultrasonic and infrared obstacle avoidance is combined, and in this process, Wi-Fi is used to realize the information exchange between each link. In addition, after receiving the food, the user has a special "confirm to take the food" button, which will not be described here.

\footnotetext{
azenghaixia@foxmail.com, ${ }^{\text {c928400653@qq.com }}$

${ }^{*}$ Corresponding author: ${ }^{b} 13477046630 @ 139 . c o m$
}

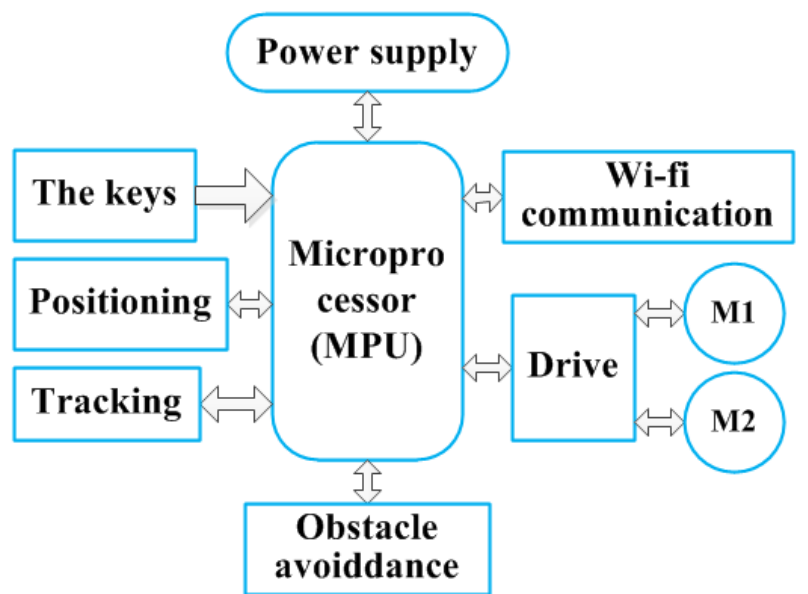

Figure 1. The overall design scheme of the system

\section{Hardware design principle and circuit}

\subsection{Positioning module}

Radio frequency identification technology is used to locate the target. Through the radio frequency module, the food delivery robot can accurately identify the electronic marks (such as cards, etc.) of the positions of multiple tables, so as to accurately locate the food delivery destination.

The electronic marks can be in the form of low-cost IC cards with storage capabilities. The serial number of each card can be designed to be unique and independent, and can be encrypted and communicated separately.

The identification of IC card by card reader is realized by electromagnetic field. In this paper, the card reader chooses MFRC522 chip, which has stable radio 
signal transmission, low power consumption and low cost, and its main frequency is $13.56 \mathrm{MHz}^{[1]}$.

\subsection{Tracking navigation}

As shown in Table 1, the performance comparison of various navigation methods currently used is listed. Considering the market feedback, path setting, feedback accuracy, anti-interference ability, cost and other factors, the infrared tracking navigation method is selected in the design of this paper ${ }^{[2]}$.

The cost of infrared navigation is low ${ }^{[3]}$. Through continuous emission of infrared ray, diffuse reflection occurs when white is encountered, and the infrared receiving tube accepts the reflected infrared ray ${ }^{[4]}$; when black is encountered, the infrared ray is absorbed and cannot receive the reflected infrared ray. The black-and-white line tracking is carried out by whether black line is received. Infrared navigation layout and adjustment is more convenient, the anti-jamming ability to the external environment is more general.

Table 1. Performance comparison of various navigation methods

\begin{tabular}{|c|c|c|c|c|c|c|}
\hline & $\begin{array}{c}\text { Market } \\
\text { feedback }\end{array}$ & $\begin{array}{c}\text { The } \\
\text { sign }\end{array}$ & $\begin{array}{c}\text { The path } \\
\text { setting }\end{array}$ & $\begin{array}{c}\text { Anti-inte } \\
\text { rference }\end{array}$ & Precision & $\begin{array}{c}\text { The } \\
\text { cost }\end{array}$ \\
\hline $\begin{array}{c}\text { Infrared } \\
\text { navigation }\end{array}$ & mature & $\begin{array}{c}\text { Need } \\
\text { to be }\end{array}$ & fixed & medium & general & low \\
\hline $\begin{array}{c}\text { Geomagnetic } \\
\text { navigation }\end{array}$ & mature & $\begin{array}{c}\text { Need } \\
\text { to be }\end{array}$ & fixed & strong & low & low \\
\hline $\begin{array}{c}\text { Visual } \\
\text { navigation }\end{array}$ & $\begin{array}{c}\text { Subject } \\
\text { to market } \\
\text { test }\end{array}$ & $\begin{array}{c}\text { Need } \\
\text { to be }\end{array}$ & fixed & weak & general & lower \\
\hline $\begin{array}{c}\text { Lidar } \\
\text { navigation }\end{array}$ & mature & $\begin{array}{c}\text { Need } \\
\text { to be }\end{array}$ & free & strong & high & high \\
\hline $\begin{array}{c}\text { Memory } \\
\text { navigation }\end{array}$ & mature & $\begin{array}{c}\text { Need } \\
\text { to be }\end{array}$ & free & stronger & low & higher \\
\hline
\end{tabular}

The infrared tracking navigation circuit designed in this paper is shown in Figure 2. The infrared transmitter of the sensor constantly emits infrared ray, when the receiving end does not receive the infrared ray, the diode is in an extinguished state; when the receiving end receives the infrared ray, the photosensitive triode is saturated. At this time, the amplifier outputs low and the diode is lit.

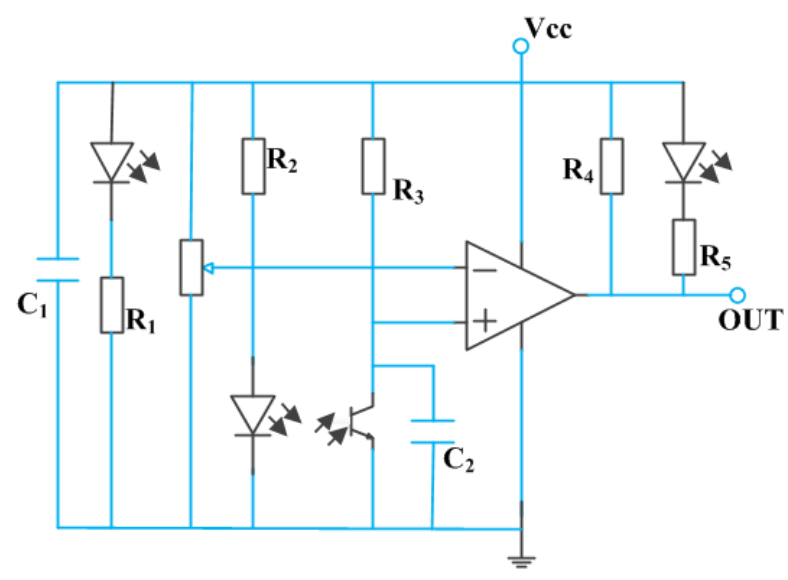

Figure 2. The infrared tracking navigation circuit

\subsection{Obstacle avoidance module}

The food delivery robot senses the changes of the external environment through sensors and responds. In this paper, the obstacle avoidance method combining ultrasonic sensor and infrared sensor is adopted. Infrared sensors can hardly identify black and transparent objects, but ultrasonic sensors can; on the contrary, the ultrasonic sensor will be affected by the shape, surface roughness and other factors of obstacles, for the relatively moving object identification accuracy is not high or unable to identify, but the infrared sensor can. Therefore, the combination of the two can improve the reliability and accuracy of the obstacle avoidance module.

\subsubsection{Ultrasonic sensor}

Ultrasonic sensors can detect the transparent material such as glass, mirror, etc. The sensor transmits ultrasonic signal, returns after encountering an obstacle, and is received by the receiving device. During this process, the timer records the duration $t$ of ultrasonic wave from sending to returning. Suppose the speed of the corresponding medium in the process of propagation is $\mathrm{v}$, then the distance of the obstacle is ${ }^{[5]}$ :

$$
\mathrm{L}=\mathrm{v} \cdot \frac{\mathrm{t}}{2}
$$

The ultrasonic module has two ports: TRIG (control terminal) and ECHO (receiver terminal). Its control timing sequence is shown in Figure 3, and its control circuit adopts the conventional design of ultrasonic sensors, which will not be described here.

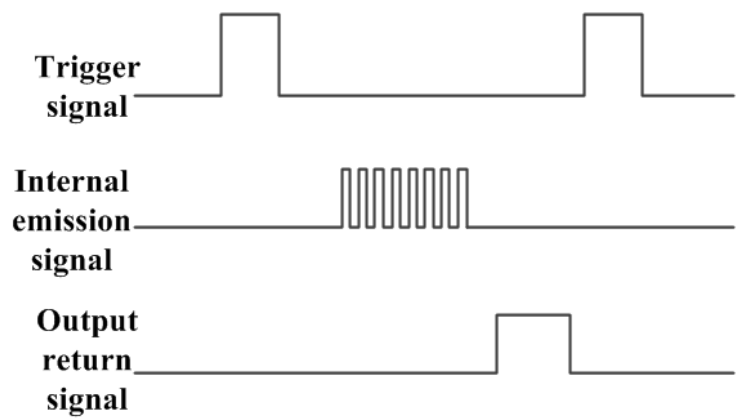

Figure 3. Ultrasonic control timing sequence

\subsubsection{Infrared sensor}

The transmitting end of the infrared sensor emits infrared ray, and the receiving end receives infrared ray. A set of infrared sensors are placed on both sides of the moving direction of the food delivery robot. When there is an object in the close range, the sensor receives strong infrared reflection and determines the obstacle ahead, so as to stop moving forward and avoid the obstacle [6]. When there is no object in the close range, the received infrared is weak, the food delivery robot judges that there is no obstacle ahead and continues to move forward.

Infrared device has the characteristics of small measuring range, short ranging distance and short response time. The disadvantage is that it is impossible to detect objects that are nearly black or transparent. 


\subsection{Drive unit}

Compared with the stepper motor, the moving parts in this design do not need too high precision, and the control of the stepper motor is relatively complex, therefore, the DC motor is selected as the moving parts, through the gear to drive two DC motors, improve the output torque, reduce the inertia of the load; and the speed of the motor is controlled by designing PWM with different duty cycle.

The motor driving circuit diagram is shown in Figure 4. The robot is controlled by DC motor with magnetic anti-interference reduction gear. Its working voltage is $5 \mathrm{~V}$ and the internal resistance of the winding is $20.4 \mathrm{ohm}$. The DC reducer motor has two input ports, when the pressure difference appears between the two ports, the motor will rotate in the positive or negative direction. The two motors are connected to the tires on both sides of the robot chassis, and the forward and reverse rotation of the motors drives the tires, in order to make the robot move forward, backward, left and right.

The driver chip provides the enabling bit and the control voltage for M1 and M2 to realize the function of forward rotation and reversal, so as to control the movement direction of the food delivery robot.

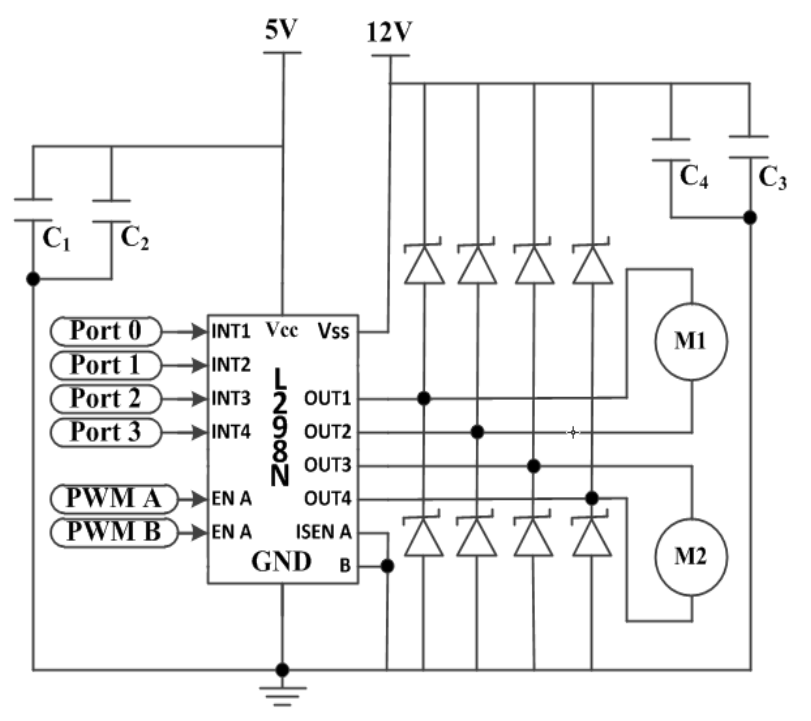

Figure 4. Motor driving circuit diagram

The motor adopts PWM principle for speed regulation. The purpose of speed regulation is achieved by adjusting the duty ratio of square wave.

The logical truth table for DC motor drive is shown in Table 2. By controlling INT1 and INT2 ports, the output of OUT1 and OUT2 can be controlled, so that the positive and negative pressure difference can be generated by the motor control signal, so as to control the positive and reverse rotation of the motor of the left tire. Similarly, by controlling INT3 and INT4 ports, the forward and reverse rotation of the motor of the right tire can also be controlled.

When $\mathrm{IN} 1=1$ and $\mathrm{IN} 2=0$, the current flows from OUT1 to OUT2, and the left motor reverses.

When $I N 1=0$ and $I N 2=1$, the current flows from OUT2 to OUT1, and the left motor is rotating positively.
When IN $3=0$ and IN4=1, the current flows from OUT4 to OUT3, and the motor on the right side reverses.

When $\mathrm{IN} 3=1$ and IN4=0, the current flows from OUT3 to OUT4, and the motor on the right is rotating positively ${ }^{[7]}$.

Table 2. The logical truth table for DC motor drive

\begin{tabular}{|c|c|c|c|}
\hline & INT1 & INT2 & \\
\hline \multirow{4}{*}{ Motor 1 } & 0 & 0 & Standby \\
\cline { 2 - 4 } & $1 / \mathrm{PWM}$ & 0 & Forward \\
\cline { 2 - 4 } & 0 & $1 /$ PWM & Reverse \\
\cline { 2 - 4 } & 1 & 1 & The brake \\
\hline \multirow{4}{*}{ Motor 2 } & INT3 & INT4 & \\
\cline { 2 - 4 } & 0 & 0 & Standby \\
\cline { 2 - 4 } & $1 / \mathrm{PWM}$ & 0 & Forward \\
\cline { 2 - 4 } & 0 & $1 / \mathrm{PWM}$ & Reverse \\
\hline
\end{tabular}

\subsection{Wi-Fi communication}

This paper uses Wi-Fi wireless communication mode for information transmission and control. It can realize RS232 serial communication, and the URXD and UTXD pins on the module are connected with the TX and RX of the microcontroller ${ }^{[8]}$.

\section{The software design}

The software program in this paper is programmed with $\mathrm{C}$ language, and the program flow chart of the control system is shown in Figure 5.

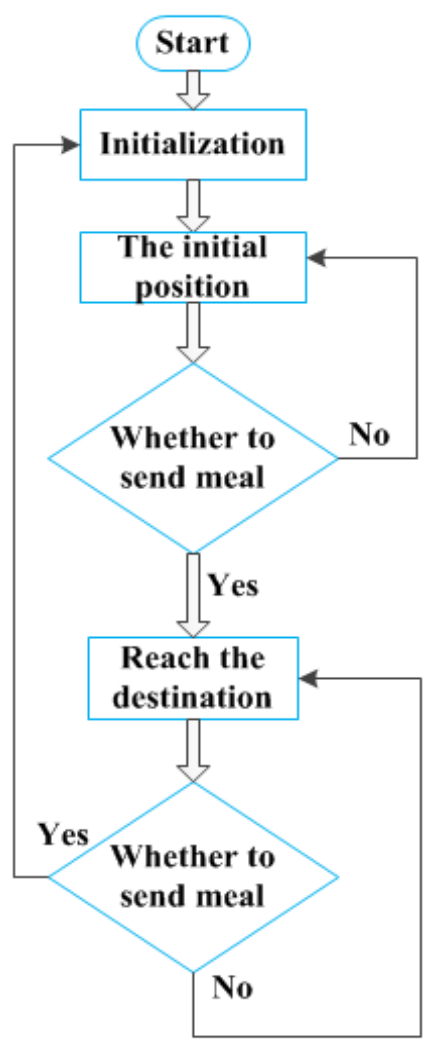

Figure 5. Program flow chart of the control system 
Using MSP430 produced by Texas Instruments as the control chip, using the 64-pin square flat package, with $48 \mathrm{~KB}+256 \mathrm{~B}$ flash memory, 2KB RAM. MSP430 series chips can work in $-40^{\circ} \mathrm{C}-85^{\circ} \mathrm{C}$ temperature environment, with ultra-low power consumption: standby mode of $1.6 \mu \mathrm{A}$, off mode of $0.1 \mu \mathrm{A}$, active mode of $280 \mu \mathrm{A}$, and has 5 power saving modes; 16-bit RISC architecture, 16-bit integrated register and constant generator, 2 built-in 16-bit timers, A fast 12-bit analog to digital converter and $48 \mathrm{I} / \mathrm{O}$ pins, etc ${ }^{[9]}$.

In addition, PWM wave adopts delay function output to control motor speed. The program flow chart of the ultrasonic module is shown in Figure 6. This part of the program is developed by interrupting. Timer T0 is used for timing, and the ultrasonic wave can be emitted by setting the $\mathrm{I} / \mathrm{O}$ port of the ultrasonic transmitter at a high level with every period of time. After the ultrasonic transmission, T0 starts the timing, and when the reflected signal is received, T0 finishes the timing, and the distance is calculated. If no return signal is received, T0 overflows the interrupt and outputs an error.

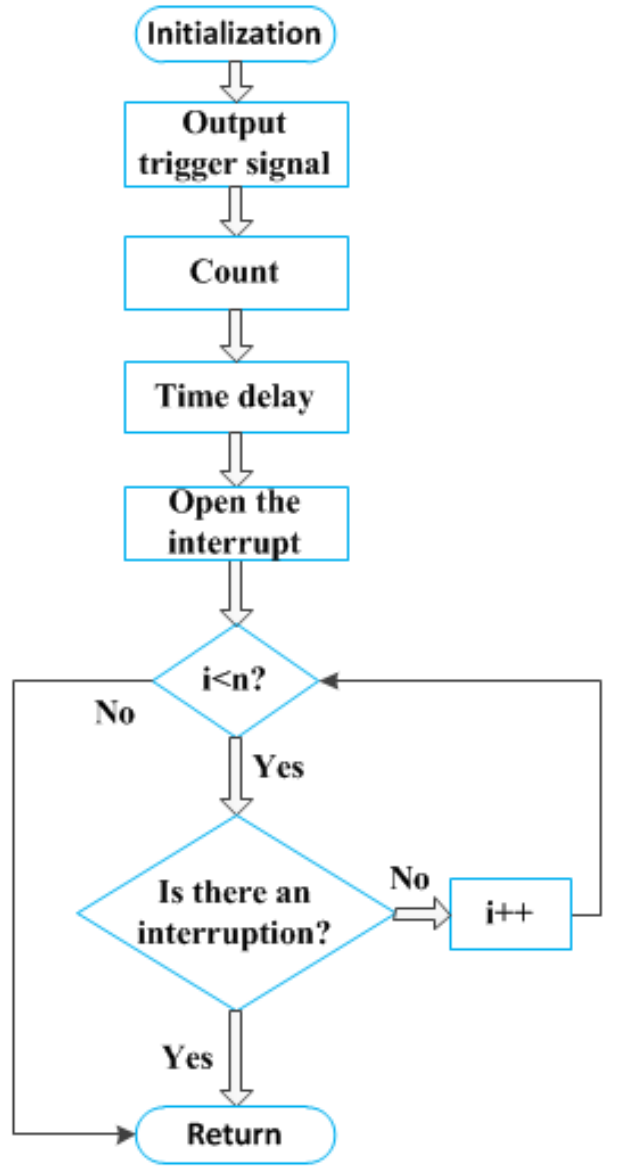

Figure 6. Program flow chart of the ultrasonic module

\section{Conclusions}

This paper presents a design of an intelligent food delivery robot control system. Through the integrated use of positioning, tracking, obstacle avoidance, communication and other modules, and in the process of robot movement, adopting PWM wave to control the speed of the motor, the food delivery robot can run smoothly in the process of food delivery, and efficiently complete the task of food delivery.

\section{Acknowledgments}

Thanks to the other two authors who participated in the design research with me and helped to complete this article. In addition, the completion of this paper also has the assistance of the relevant students, and I would like to thank them.

\section{References:}

1. Zhang J, Zhang JW. (2015) Robot operating system. Tsinghua University Press, Beijing.

2. Hu H, Fan Y, Li DJ. (2017) Research and Design of Intelligent Food Delivery Robot. Internal combustion engine and accessories, 21: 6-8.

3. Chen YY, Cai JX, Liu ZW. (2014) Design and implementation of an intelligent tracking robot based on multipath recognition. Measurement and control technology, 33: 65-68.

4. Li QY. (2017) Research on Structure Design and Motion Control of Hotel Service Robot. Southwest Jiaotong University, Chengdu.

5. Yuan YY, Song TL. (2017) Research and design of food delivery service robot. Science and Technology Innovation and Application, 04: 62.

6. Su YH, Ren J. (2018) Intelligent food delivery robot design. Science and Technology Innovation and Application, 7: 32-34.

7. Zhou W. (2020) Intelligent food delivery robot design. WenHua College, Wuhan.

8. Zhang C, Zhang GQ. (2018) Design and research of food delivery robot. Electronic manufacture, 11: 38-40.

9. Chen K. (2017) Design and Implementation of Intelligent Food Delivery Robot Control System. Hangzhou Dianzi University, Hangzhou. 